\title{
Hot Spot Effect of Optical Nanoantenna to Enhance Localized Photothermal Conversion
}

\author{
Yatao Ren, ${ }^{1,2, \dagger}$ Qin Chen, ${ }^{1,2, \dagger}$ Hong Qi ${ }^{1,2, *}$ and Liming Ruan ${ }^{1,2, *}$
}

Plasmonic nanoparticles induced localized heating has drawn much attention due to its current and potential applications like solar energy harvesting and storage, photothermal therapy, nanosurgery, nanochemistry, optofluidics, and surface-enhanced Raman spectroscopy. Ultilizing a nanoparticle or other sorts of nanostructures under the illumination of strong monochromatic light can create a localized nanoscale source of heat with minimum influence on its surrounding area. In the present work, we studied quantitatively the amplification effect of a nanosphere dimer on the hot spot located in the interparticle gap. It is found that in the nanotrimer system under consideration, the thermal amplification efficiency increases with the increasing of the size of amplifier, under the illumination of monochromatic light. However, high localized photothermal conversion efficiency does not necessary mean high local temperature, since the local temperature rise is induced by two independent effects, namely, thermal superposition effect and plasmonic coupling effect. The contribution of these two effects on the temperature rise of the hot spot is also investigated. At last, we discussed the possibility of using the hot spot effect on the heat-assisted magnetic recording system to reduce the size and complexity of the writing head by replacing the gold nanoheater with metal nitrides.

Keywords: Optical nanoantenna; hot spot effect; photothermal conversion; thermoplasmonics

Received 13 December 2018, Accepted 15 January 2019

DOI: $10.30919 /$ esee $8 \mathrm{c} 178$

\section{Introduction}

Recently, plasmonic nanoparticles induced localized heating has drawn much attention due to its current and potential applications like solar energy harvesting and storage, ${ }^{1-5}$ photothermal therapy, ${ }^{6-11}$ nanosurgery, ${ }^{12,} 13$ nanochemistry, ${ }^{14-16}$ optofluidics, ${ }^{17}$ and surface-enhanced Raman spectroscopy. ${ }^{18-20}$ In addtion, a newly proposed magnatic storage technique named heat-assisted magnetic recording (HAMR) can greatly improve the data capacity of hard drives by storing the same amount of information to a much smaller region. However, this is extremely difficult to achieve since it requires a very small writing head to be ultilized to generate a severe temperature rise. ${ }^{21}$ With the development of thermoplasmonics, this problem can be solved by ultilizing a nanoparticle or other sorts of nanostructures under the illumination of strong monochromatic light, which can create a localized nanoscale source of heat with minimum influence on its surrounding media.

An isolated small nanoparticle will generate a much lower temperature increase compared to other larger nanoparticles under the same situation, ${ }^{22}$ which obeys $\Delta T_{\max } \propto R_{N P .}^{2} .^{23,24}$ It means that to obtain a certain level of temperature rise, higher laser intensity is required when we try to reduce the particle size. However, this will bring in other new issues, since higher laser intensity will, at the same time, heat other parts of the

'School of Energy Science and Engineering, Harbin Institute of Technology, Harbin 150001, P. R. China

${ }^{2}$ Key Laboratory of Aerospace Thermophysics, Ministry of Industry and Information Technology, Harbin 150001, P. R. China

*E-mail:qihong@hit.edu.cn; ruanlm@hit.edu.cn

"These two authors contribute equally to this work. whole system. In addition, thermal diffusion makes it even harder to maintain a high temperature rise in a localized region, since it tends to produce a spatially uniform temperature. To avoid this, the local electromagnetic field needs to be enahnced to improve the localized photothermal conversion efficiency. Govorov and coworkers ${ }^{22,23}$ studied systematicly the amplification effect of nanoparticle dimers (amplifier) on the nearby smaller nanoparticles (nanosphere and nanorod). It was found that the collective effect in manybody system of nanoparticles can be utilized to strongly amplify the heating effect and create a localized hot spot. Toroghi et al..$^{25}$ reported a dramatic enhancement of the thermally induced optical response in compositionally heterogeneous plasmonic trimers. They found that the electromagnetic field around a silver nanoparticle dimers under pulsed illumination can be enhanced by more than two orders of magnitude by adding a relatively small gold nanoparticle in the dimer gap. The ultimate goal of studies concerning the hot sopt effect is to manipulate the nanosacale localized temperature for current and potential applications. Baldwin et $a l .^{26}$ illustrated that the temperature change can be both localized and controllable through a Fano interference. They concluded that the nanoscale temperature change can be manipulated by Fano resonnace with carefully chosen composition and morphology of nanoparticles. As mentioned above, a very interesting and promissing application is information storage using HAMR. Chalener et $a l^{27}$ developed a nanoscale optical spot for HAMR that significantly improves the storage density of hard drives. The system they developed can be applied to transfer optical energy to a lossy metallic medium and remain confined in a spot much smaller than the diffraction limit, which can be used to heat a $70 \mathrm{~nm}$ track. By using the nanoamplifier system instead, this dimension may be further reduced.

Despite that, the mechanism of the above mentioned amplification 
effect still needs to be investigated in order to predict and regulate precisely the temperature rise of the hot spot, therefore to improve the performace of localized nanoheater system. In the present work, we studied quantitatively the amplification effect of a nanosphere dimer on the hot spot located in the interparticle gap. The contribution of thermal superposition effect and plasmonic coupling effect on the temperature rise of the hot spot is also investigated. Finally, we discussed the possibility of using the hot spot effect on the HAMR system to reduce the size and complexity of the writing head.

\section{Theory and Methods}

We consider a heat transfer process in an infinite space with nanoparticles under continuous illumination of monochromatic light. The heat transfer equation can be expressed $\mathrm{as}^{28}$ :

$\left\{\begin{array}{l}\rho_{\mathrm{p}} c_{\mathrm{p}} \partial_{t} T(\mathbf{r}, t)-k_{\mathrm{p}} \nabla^{2} T(\mathbf{r}, t)=q(\mathbf{r}), \quad \text { inside nanostructure } \\ \rho_{\mathrm{w}} c_{\mathrm{w}} \partial_{t} T(\mathbf{r}, t)-k_{\mathrm{w}} \nabla^{2} T(\mathbf{r}, t)=0, \quad \text { outside nanostructure }\end{array}\right.$

where subscripts ' $\mathrm{p}$ ' and ' $\mathrm{w}$ ' represent plasmonic nanoparticle and water, respectively. $\rho, c$, and $k$ are the density, heat capacity, and thermal conductivity, respectively.

The heat generation rate $q(\mathbf{r})$ can be expressed $\mathrm{as}^{28}$ :

$$
q(\mathbf{r})=\frac{\omega}{2} \operatorname{Im}\{\varepsilon(\mathbf{r}, \omega)\}|\mathbf{E}(\mathbf{r})|^{2}
$$

where $\omega$ is the frequency of laser and $\varepsilon$ is dielectric constant at position r. E(r) is the local electric field intensity, which can be obtained by solving the following equation ${ }^{29}$ :

$$
\nabla \times \mu_{\mathrm{r}}^{-1}[\nabla \times \mathbf{E}(\mathbf{r})]-k_{0}^{2}\left(\varepsilon_{\mathrm{r}}-j \frac{\sigma}{\omega \varepsilon_{0}}\right) \mathbf{E}(\mathbf{r})=0
$$

where $\mu_{\mathrm{r}}, \varepsilon_{r}$, and $\sigma$ are the relative permeability, permittivity, and conductivity, respectively. $k_{0}$ denotes the wave number.

In the present work, the above equations are solved by Comsol Multiphysics, which is based on finite element method. The maximum element size inside the nanoparticles are set as $5 \mathrm{~nm}$. The meshes in other computational domain are automatically generated. For the solution of electric field, the boundary conditions are set as perfectly matched layer. For the solution of temperature field, the boundary conditions are set as a constant temperature of $300 \mathrm{~K}$. The verification of the numerical model are provided in the Supporting Information. In the following content, the refractive index of the surrounding media is set as 1.33 , which is similar to water. The thermal properties of gold nanoparticles are regarded as the same as bulk metal ${ }^{30}$, without considering size effect. the thermal conductivity of gold and water are set as $317^{31}$ and $0.6^{31} \mathrm{Wm}^{-1} \mathrm{~K}^{-1}$, respectively.

\section{Results and Discussion}

\subsection{Gold nanoheater with symmetrical amplifier}

The optical nanoantenna system under consideration is illustrated in Fig. 1a. The polarization direction of incident light is along the axis of the nanotrimer. The relatively smaller nanosphere located between a nanosphere dimer acts like a nanoheater to perform a much more distinct thermal effect. To quantify the thermal amplification of the nanosphere heater, the thermal amplification efficiency is defined as follows (a)
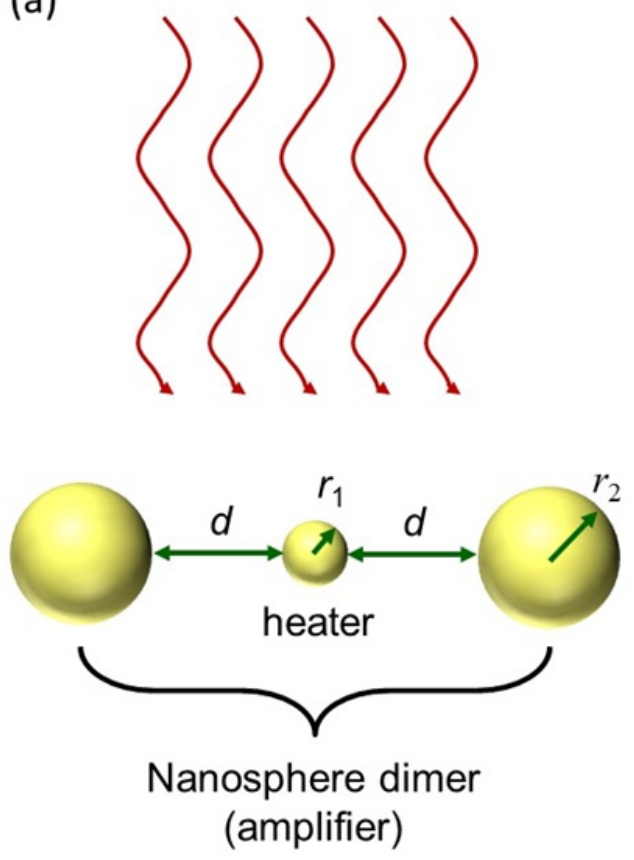

(b)

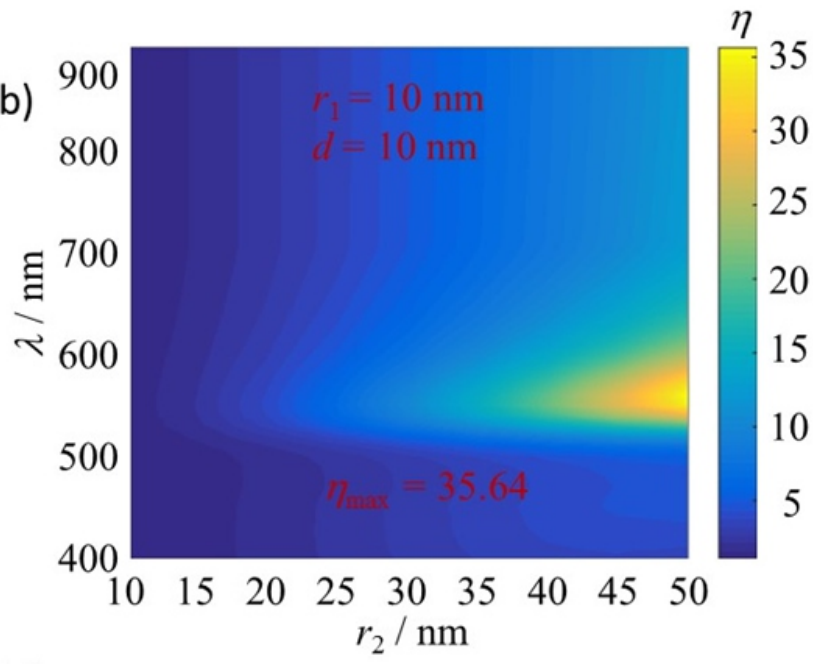

(c)

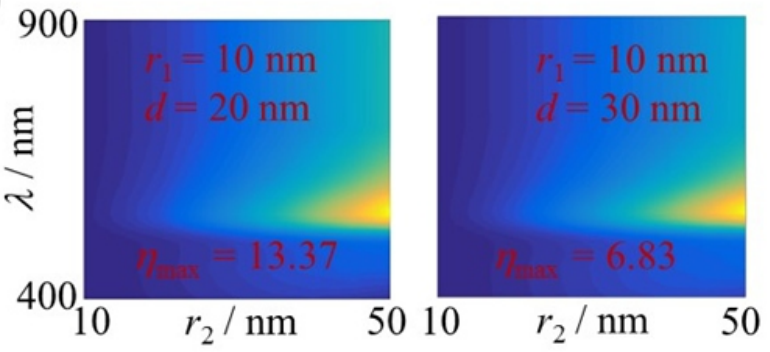

Fig. 1 Hot spot effect of gold nanoantenna. a) Schematic of the system under investigation. The polarization direction of incident light is along the axis of the nanotrimer. b) Thermal amplification efficiency of nanosphere heater $\left(r_{1}=10 \mathrm{~nm}\right)$ with $d=10 \mathrm{~nm}$ under different radius $r_{2}$ of nanosphere dimers. c) Thermal amplification efficiency of nanosphere heater $\left(r_{1}=10 \mathrm{~nm}\right)$ with $d=20 \mathrm{~nm}$ and $30 \mathrm{~nm}$. 


$$
\begin{gathered}
\eta=\frac{q_{\text {heater }}^{\mathrm{v}}}{q_{\text {aver }}^{\mathrm{v}}}=\frac{I \cdot C_{\text {heater }}^{\text {abs }} / V_{\text {heater }}}{I \cdot C_{\text {all }}^{\mathrm{abs}} / V_{\text {all }}}=\frac{C_{\text {heater }}^{\text {abs }}\left(V_{\text {antenna }}+V_{\text {heater }}\right)}{V_{\text {heater }}\left(C_{\text {heater }}^{\text {abs }}+C_{\text {antenna }}^{\text {abs }}\right)} \\
=\frac{C_{\text {heater }}^{\text {abs }}\left(2 \cdot r_{\text {antenna }}^{3}+r_{\text {heater }}^{3}\right)}{r_{\text {heater }}^{3}\left(C_{\text {heater }}^{\text {abs }}+C_{\text {antenna }}^{\text {abs }}\right)}
\end{gathered}
$$

where the subscript 'all', 'aver', 'antenna', and 'heater' stand for the properties of nanoparticle trimer, average of nanoparticle trimer, nanoparticle dimer, and the heater, respectively. $I$ is the light intensity. $C, V$, and $r$ represent absorption cross section, volume, and radius, respectively.

The thermal amplification efficiency $\eta$ can be utilized as an indicator to evaluate the thermal amplification performance of the nanosphere dimer. For the radius of heater $r_{1}=10 \mathrm{~nm}$, the value of $\eta$ corresponding to different $r_{2}$ and $d$ are illustrated in Fig. $1 \mathrm{~b}$ and 1c. It can be seen that for different interparticle distance $d$, the variation trend of $\eta$ corresponding to $\lambda$ and $r_{2}$ are almost the same, without considering its absolute value. However, the maximum value of $\eta$ decreases dramatically with the increasing of interparticle distance $d$, which is due to the reduction of plasmonic coupling effect. Therefore, one can enhance the localized optothermal conversion efficiency by simply reducing the interparticle distance. Moreover, from Fig. 1b, it can be seen that the thermal amplification efficiency increases with the increasing of the size of amplifier, under the illumination of monochromatic light. This is meaningful for applications that requires very high temperature in a localized spot. However, high localized photothermal conversion efficiency does not necessary mean high local temperature, since the local temperature rise is induced by two independent effects, namely, thermal superposition effect (TSE) and plasmonic coupling effect $(\mathrm{PCE})^{32}$. TSE refers to the enhanced thermal accumulation induced by the heat transfer from nearby hot nanoparticles, while PCE refers to the thermal accumulation induced by the plasmonic coupling of nearby nanoparticles, which lead to the enhancement of local electromagnetic field and heat generation. In Figs. 2(a-c), we show the maximum temperature rise and absorption cross section of the nanoheater. The blue bar stands for the maximum temperature rise considering both thermal superposition effect and plasmonic coupling effect, while the red bar shows the results that only consider the thermal superposition effect, which means the heat generation rate in this situation is set as the same as an isolated nanoparticle. It can be seen that the maximum temperature rise considering both TSE and PCE (blue bar) is not always increasing with the increase of the size of the amplifiers $\left(r_{2}\right)$, which is consistent with trends of the absorption cross section of the nanoheater $\left(C_{\text {abs, heater }}\right)$. This strongly indicates that the amplification effect does not necessary being enhanced with the increasing of $r_{2}$ and PCE dominates in the heating
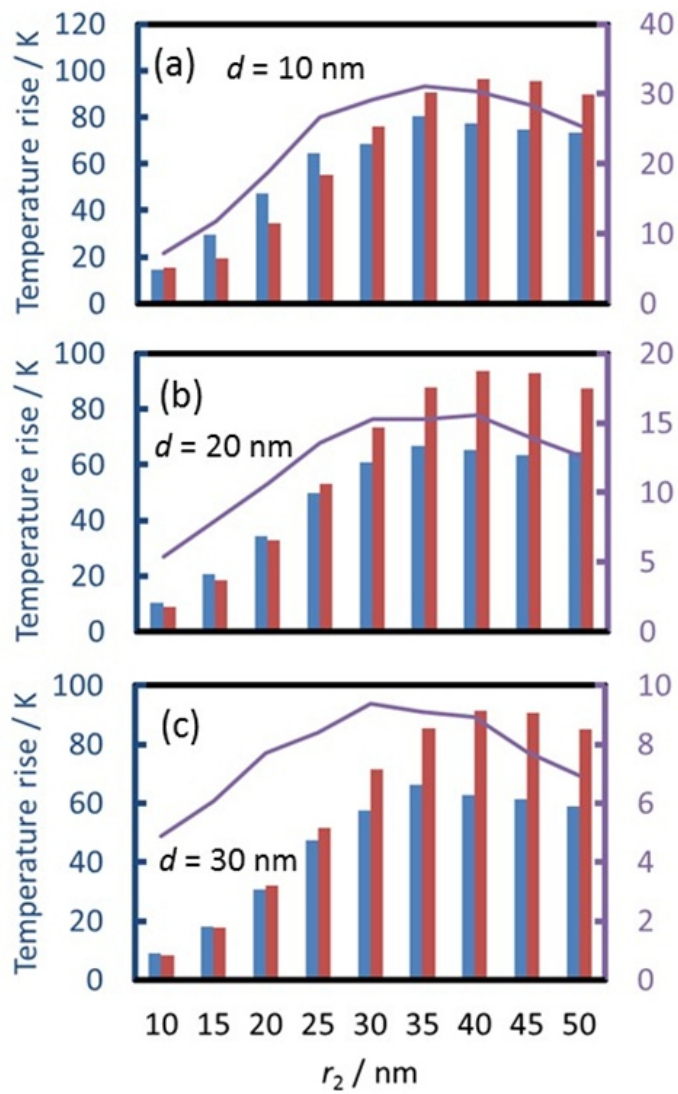

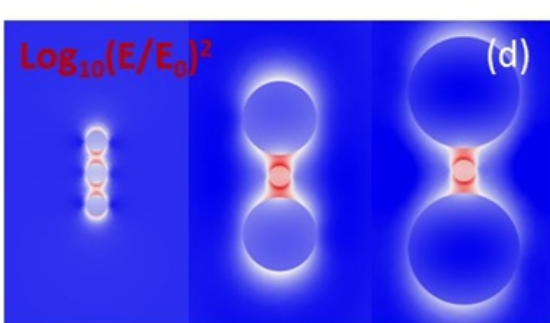

$r_{2}=10 \mathrm{~nm} \quad r_{2}=35 \mathrm{~nm} \quad r_{2}=50 \mathrm{~nm}$ $d=10 \mathrm{~nm} \quad d=10 \mathrm{~nm} \quad d=10 \mathrm{~nm}$
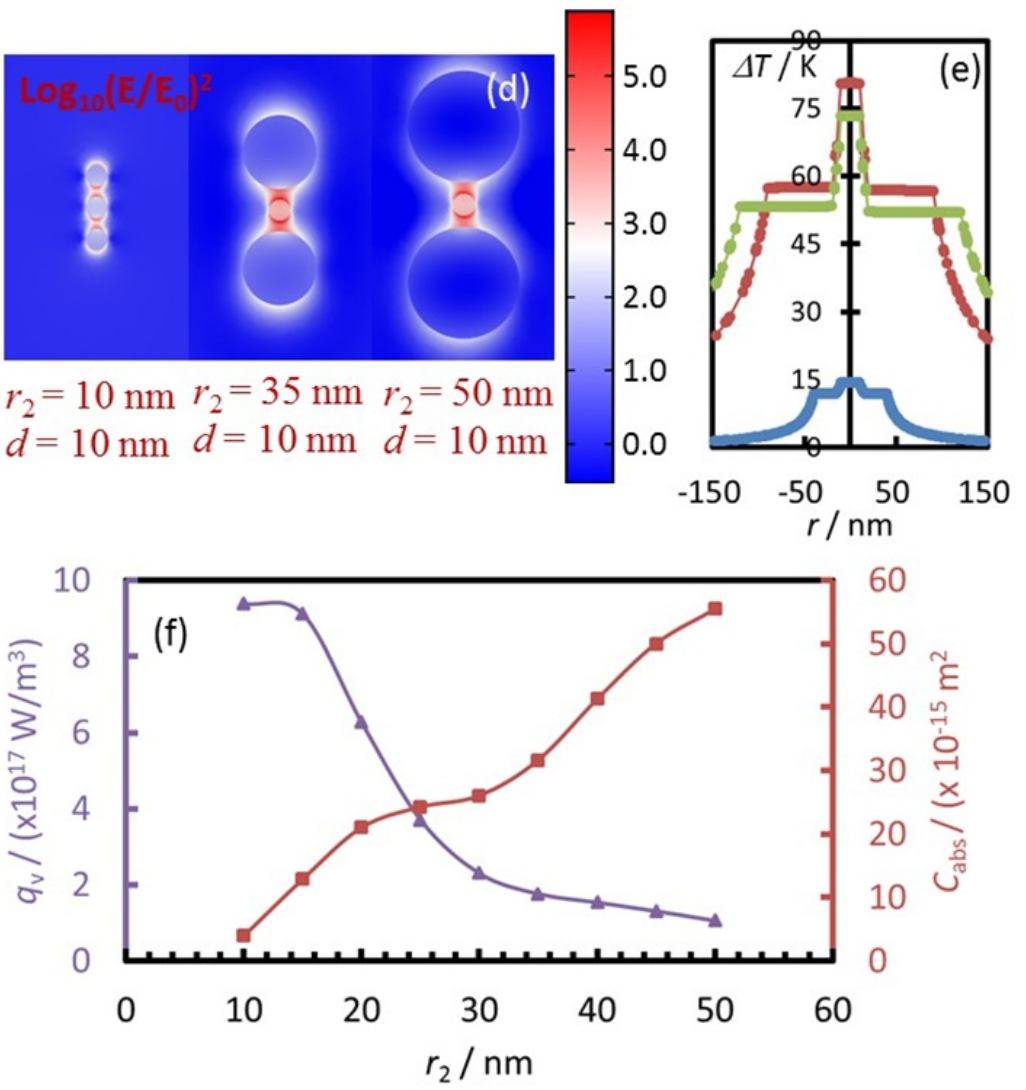

60

50 है

40

$30 \stackrel{-}{\circ}$

20

10

0

Fig. 2 Thermal superposition effect and plasmonic coupling effect in nanoparticle trimer. Maximum temperature rise in nanoparticle trimer and absorption efficiency of nanoheater when the interparticle distances are (a) $d=10 \mathrm{~nm}$; (b) $d=20 \mathrm{~nm}$; and (c) $d=30 \mathrm{~nm}$, where the incident wavelength and intensity are $532 \mathrm{~nm}$ and $1.0 \times 10^{9} \mathrm{~W} / \mathrm{m}^{2}$. The blue bar stands for the maximum temperature rise considering both thermal superposition effect and plasmonic coupling effect, while the red bar only considers the thermal superposition effect, which means the heat generation rate in this situation is set as the same as an isolated nanoparticle. (d) Electric field enhancement for different sizes of nanoparticle dimer. (e) Temperature profiles for nanoparticle trimers corresponding to different sizes of $r_{2}$ corresponding to the scenarios in Fig. 2d. (f) Heat generation rate and absorption cross section of an isolated nanoparticle. 
process of nanoparticles trimers. It is worth noting that for most cases the maximum temperature rise when only TSE is considered is higher than those of considering both PCE and TSE, especially for larger $r_{2}$, which means for nanotrimer system considered in the present work, the photothermal conversion of the amplifier is strongly suppressed while at the same time that of the nanoheater is strongly enahnced (Figs. $2 \mathrm{~d}$ and 2e). Also it can be seen from Fig. 2e that when both the PCE and TSE are taken into consideration, the maximum temperature rise always appears at the nanoheater. However, when the PCE is not taken into consideration, the maximum temperature rise occurs at the amplifier (Fig. 3). This is consistent with the results in other references that an isolated small nanoparticle will generate a much lower temperature increase compared to other larger nanoparticles. Moreover, it is very interesting to note that the maximum temperature rise is also not positive correlated with the size of the amplifier even when plasmonic coupling effect is not considered, which is confusing since the absorption cross section of an isolated nanoparticle increases with the increasing of $r_{2}$ (Fig. 2f). The reason is that the maximum temperature rise of a sphere with internal heat generation is decided by a function of the particle radius and the heat generation rate, which is as follows (refer to supporting information for details)

$$
\Delta T=\frac{q_{\mathrm{v}} R^{2}}{6 k_{\mathrm{Au}}}+\frac{q_{\mathrm{v}} R^{2}}{3 k_{\mathrm{w}}}
$$

where $R$ is the radius of the nanoparticle. As shown in Fig. $2 \mathrm{f}$, with the increasing of particle size, the heat generation rate $q_{\mathrm{v}}$ decreases, leading to the overall decrease of temperature rise.

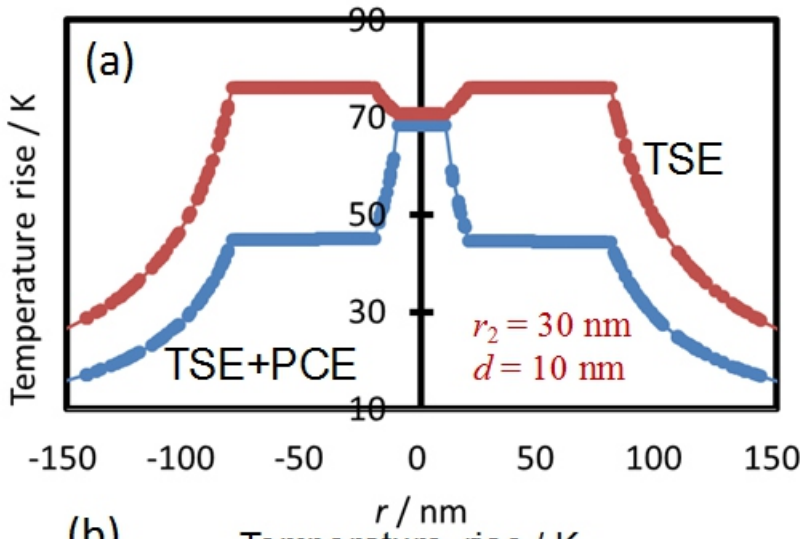

(b) Temperature rise / $\mathrm{K}$
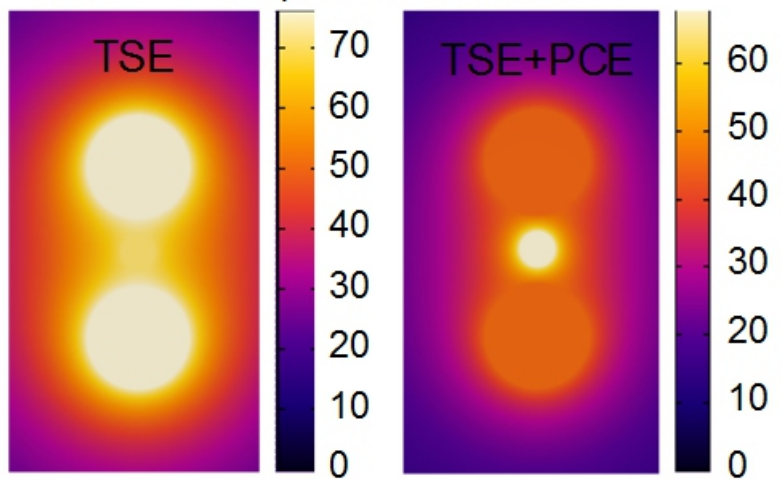

Fig. 3 The temperature response of thermal superposition effect and plasmonic coupling effect. (a) Temperature distribution along the axis of the nanoparticle dimer. (b) Temperature distribution when considering PCE and not considering PCE.

\subsection{Gold nanoheater with asymmetrical amplifier}

To investigate the influence of the asymmetrical amplifier on the light and heat transfer in the nanotrimer system, we changed the size of one nanoparticle in the amplifier and keep that of another as constant (see Fig. 4a). The nanoparticle with variable sizes is named as regulator. Fig. $4 \mathrm{~b}$ illustrates the maximum temperature rise of three nanoparticles (heater, regulator, and amplifier). The polarization direction of incident light is along the axis of the nanotrimer. It can be seen that when the size of the regulator is identical to that of the amplifier, the highest temperature rise can be obtained, which also can be seen in Fig. 4c. Moreover, in the nanotrimer system, the bigger one between the regulator and amplifier always has a higher temperature rise. For example, when the radius of regulator is $25 \mathrm{~nm}$ (smaller than that of the amplifier, which is $35 \mathrm{~nm}$ ), the temperature of regulator is lower than that of the amplifier. Meanwhile, when the radius of the regulator is 60 $\mathrm{nm}$ (larger than that of the amplifier), it is the other way around (see Fig. 4d).

\subsection{Metal nitrides nanoheater}

Metal nitrides (MNs) have been proved to be promising materials for applications in thermoplasmonics due to their high sustainability to temperature. ${ }^{33}$ The melting point of MNs is much higher than that of gold, which are $3203 \mathrm{~K}, 3225 \mathrm{~K}$, and $1337 \mathrm{~K}$ for TiN, ZrN, and $\mathrm{Au}$, respectively $^{21}$. For application like HAMR, the temperature of the nanostructures may be higher than the melting point of gold, which makes gold inapplicable in those situations, despite its outstanding optical properties. Therefore, in this section, we investigated the possibility of replacing the nanoheater with MNs to increase the thermal resistance of the nanotrimer system (see Fig. 5a). The real and imaginary part of permittivity of $\mathrm{TiN}, \mathrm{ZrN}$, and $\mathrm{Au}$ are shown in Figs. $5 \mathrm{~b}$ and $5 \mathrm{c} .^{33}$ To evaluate the performance of the Au-MN-Au nanotrimer system, we first calculated the absorption cross section of the heater and amplifier with different materials of the heater (see Fig. 5d). It can be seen that the absorption across sections of the amplifier does not have too much differences whether the material of the heater is $\mathrm{Au}$, TiN, or $\mathrm{ZrN}$, which is reasonable since the material of the amplifier remains the same and the size of the amplifier is much larger than that of the heater. For the same reason, the obvious variation of absorption cross section of the heater can be observed. Compared to $\mathrm{Au}$, the localized surface plasmon resonance wavelength of MNs nanoheater has a slightly red shift. Also, the resonance peak is getting broader, which is more obvious for TiN. However, the distribution profiles are basically the same. Also, the maximum absorption cross sections are on the same level, which means we just need to change the incident wavelength to obtain the maximum temperature rise.

Therefore, TiN is applied as an example. In the following cases, the material of nanoheater is set as TiN. Fig. 5e shows the highest temperature of the heater (TiN) and amplifier (Au) under the illumination of light in different intensities. It is obvious that the difference between the maximum temperature of TiN and $\mathrm{Au}$ increase with the increasing of laser intensity. Before $\mathrm{Au}$ is heated up to its melting point $(1337 \mathrm{~K})$, the temperature of nanoheater TiN can reach about $1555 \mathrm{~K}$, which can totally satisfy the required operating temperature of HAMR. Similar to the case of Au nanotrimer, the amplification effect of the Au nanodimer is also highly related to the size of the amplifier. Fig. $5 \mathrm{f}$ illustrates the temperature distribution in the Au-TiN-Au nanotrimer system with different $r_{2}$. It can be seen that the temperature rise of the TiN heater is not linearly related to $r_{2}$. For the simulated cases, when $r_{2}$ equals to $35 \mathrm{~nm}$, the temperature of TiN heater reaches its maximum, which means the size of the amplifier 
(a)<smiles>CCCCCCCCCCCCCCCCCCCCCCCCCCCCCCC</smiles>

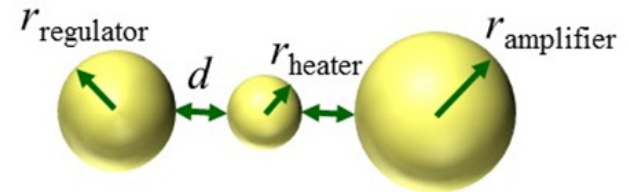

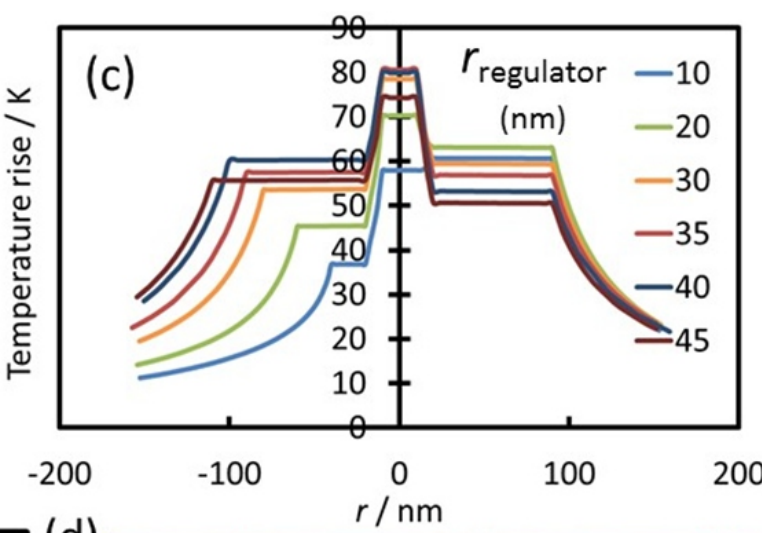

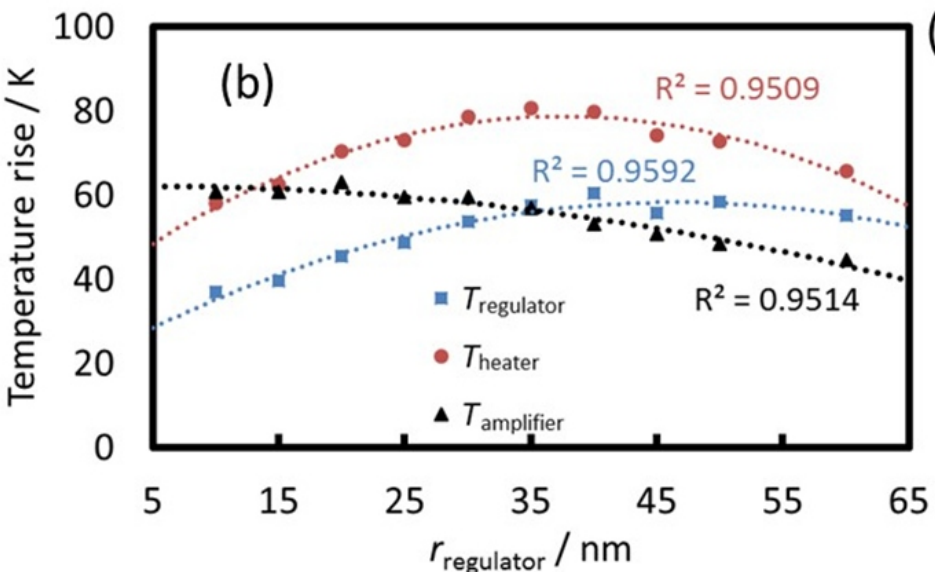

(d)
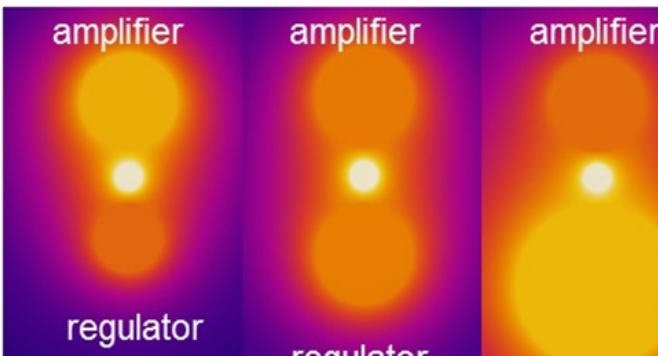

regulator

regulator

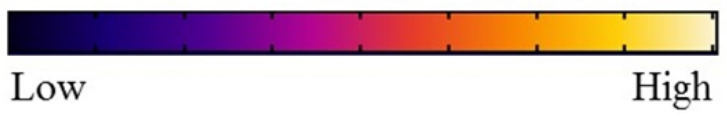

Fig. 4 The thermal effect of nanoheater with asymmetrical amplifier. (a) Schematic of the system under investigation. The nanoparticle with variable sizes is named as regulator. The polarization direction of incident light is along the axis of the nanotrimer. (b) The maximum temperature rise of the three nanoparticles (regulator, heater, and amplifier), where the incident wavelength and intensity are $532 \mathrm{~nm}$ and $1.0 \times 10^{9} \mathrm{~W} / \mathrm{m}^{2}$. The interparticle distance is set as $d=10 \mathrm{~nm}$. The radius of heater and amplifier are set as $r_{\text {heater }}=10 \mathrm{~nm}$ and $r_{\text {amplifer }}=35 \mathrm{~nm}$, respectively. (c) Temperature profile of the nanoparticle trimer along its axis with different sizes of regulator. (d) Temperature distributions of nanoparticle trimer for $r_{\text {reglator }}=25,35,60 \mathrm{~nm}$.

(a)
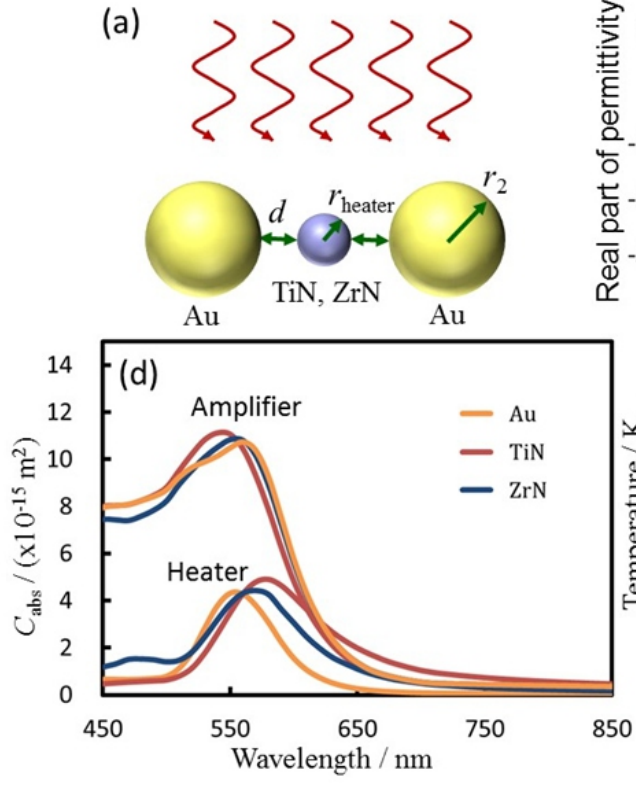
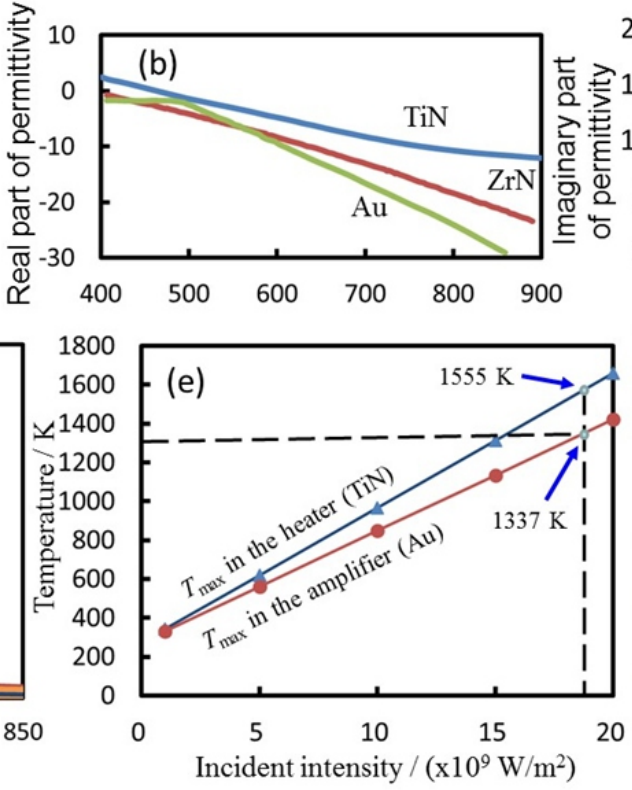
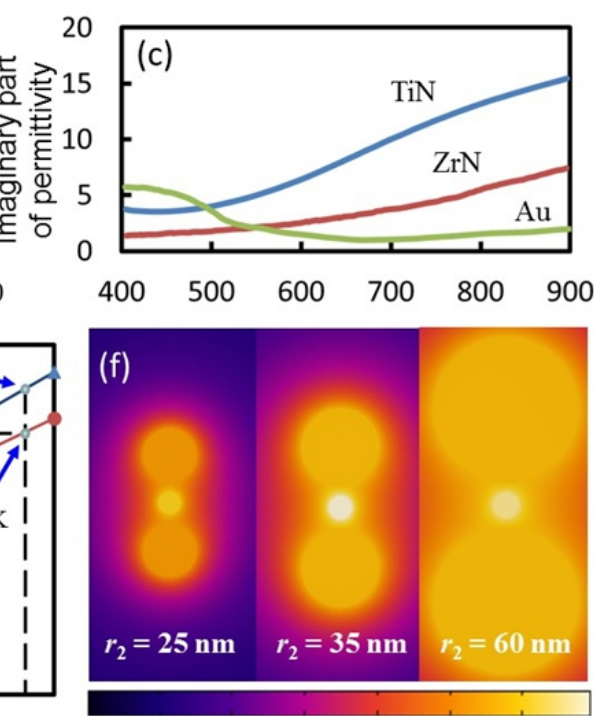

0
$200 \quad 400 \quad 600 \quad 800 \quad 1000 \quad 1200$

Temperature rise / $\mathrm{K}$

Fig. 5 Hot spot effect of gold nanoantenna with metal nitrides as nanoheater (TiN/ZrN). (a) Schematic of the system under investigation. (b) and (c) real and imaginary parts of the permittivity of $\mathrm{Au}, \mathrm{TiN}$, and $\mathrm{ZrN}$. (d) Absorption cross section of heater and amplifier in nanotrimer system, when the material of heater is $\mathrm{Au}, \mathrm{TiN}$, and $\mathrm{ZrN}$. The radius of the heater and amplifier are $10 \mathrm{~nm}$ and $35 \mathrm{~nm}$ respectively. The interpartical distance is $10 \mathrm{~nm}$. (e) Highest temperature of the heater ( TiN) and amplifier $(\mathrm{Au})$ under the illumination of light in different intensity. The radius of the heater and amplifier are $10 \mathrm{~nm}$ and $35 \mathrm{~nm}$ respectively. (f) Temperature distribution in nanotrimer system with light intensity of $2.0 \times 10^{10} \mathrm{~W} / \mathrm{m}^{2}$. 
should be carfully chosen.

\section{Conclusion}

In the present work, we studied quantitatively the amplification effect of a nanosphere dimer on the hot spot located in the interparticle gap. The contribution of thermal superposition effect and plasmonic coupling effect on the temperature rise of the hot spot is also investigated. It is found that in the nanotrimer system under consideration, the thermal amplification efficiency increases with the increasing of the size of amplifier, under the illumination of monochromatic light with a constant wavelength. However, high localized photothermal conversion efficiency does not necessary mean high local temperature, since the local temperature rise is induced by two independent effects, namely, thermal superposition effect and plasmonic coupling effect. On this basis, the case of asymmetrical amplifier is investigated. It was found that symmetrical amplifier always creates the highest temperature rise in the hot spot. At last, we discussed the possibility of using the hot spot effect on the HAMR system to reduce the size and complexity of the writing head. By replacing the nanoheater with MNs, the proposed hot spot configuration can totally create a localized high temperature without melting the amplifier.

\section{Supporting Information}

The theoretical basis for maximum temperature rise calculation of an isolated nanoparticle and the verification of the numerical model are available in Supporting Information.

\section{Acknowledgements}

This work is supported by the National Natural Science Foundation of China (No. 51806047), the Fundamental Research Funds for the Central Universities (No. HIT.NSRIF.2019064), and the China Postdoctoral Science Foundation Grant (No. 2018M630351).

\section{References}

1. W. An, J. Li, J. Ni, R. A. Taylor and T. Zhu, Energ. Convers. Manage., 2017, 151, 23-31.

2. W. An, J. Wu, T. Zhu and Q. Zhu, Appl. Energ., 2016, 184, 197-206.

3. O. Neumann, A. S. Urban, J. Day, S. Lal, P. Nordlander and N. J. Halas, ACS Nano, 2013, 7, 42-49.

4. J. Tan, Y. Xie, F. Wang, J. Lin and L. Ma, Int. J. Heat Mass Tran., 2017, 115, 1103-1112.

5. H. Liang, F. Wang, D. Li, J. Zhu and J. Tan, Int. J. Heat Mass Tran., 2019, 128, 668-678.

6. Q. Chen, Y. Ren, H. Qi and L. Ruan, J. Quant. Spectrosc. Ra., 2018, 212, 1-9.

7. M. R. Ali, M. A. Rahman, Y. Wu, T. Han, X. Peng, M. A. Mackey, D. Wang,
H. J. Shin, Z. G. Chen, H. Xiao, R. Wu, Y. Tang, D. M. Shin and M. A. ElSayed, Proc. Natl. Acad. Sci., 2017, 114, E3110-E3118.

8. Y. Ren, H. Qi, Q. Chen and L. Ruan, Int. J. Heat Mass Tran., 2017, 106, $212-$ 221.

9. Y. Ren, Q. Chen, H. Qi and L. Ruan, Nanomaterials, 2017, 7, 416.

10. Y. Huang, S. He, W. Cao, K. Cai and X. J. Liang, Nanoscale, 2012, 4, $6135-$ 6149 .

11. Y. Ren, Q. Chen, H. Qi, R. Liming and J. Dai, Int. J. Heat Mass Tran., 2018, 127, 244-252.

12. G. D. Jeffries, J. S. Edgar, Y. Zhao, J. P. Shelby, C. Fong and D. T. Chiu, Nano Lett., 2007, 7, 415-420.

13. S. Su, X. Zuo, D. Pan, H. Pei, L. Wang, C. Fan and W. Huang, Nanoscale, 2013, 5, 2589-2599.

14. G. Chen, I. Roy, C. Yang and P. N. Prasad, Chem. Rev., 2016, 116, 28262886.

15. Y. Yuan, Z. Ruan, X. Huang, Y. Jiang and H. Tan, J. Catal., 2017, 348, 246255.

16. C. Jack, A. S. Karimullah, R. Tullius, L. K. Khorashad, M. Rodier, B. Fitzpatrick, L. D. Barron, N. Gadegaard, A. J. Lapthorn, V. M. Rotello, G. Cooke, A. O. Govorov and M. Kadodwala, Nat. Commun., 2016, 7, 10946.

17. Y. Ren, H. Qi, Q. Chen, Y. Li and L. Ruan, Int. J. Heat Mass Tran., 2019, 133, 1019-1025.

18. F. De Nicola, N. S. P. Purayil, D. Spirito, M. Miscuglio, F. Tantussi, A. Tomadin, F. De Angelis, M. Polini, R. Krahne and V. Pellegrini, ACS Photonics, 2018, 5, 2418-2425.

19. H. Cho, S. Kumar, D. Yang, S. Vaidyanathan, K. Woo, I. Garcia, H. J. Shue, Y. Yoon, K. Ferreri and H. Choo, ACS Sensors, 2018, 3, 65-71.

20. H. X. Shen, W. J. Zou, Z. L. Yang, Y. X. Yuan, M. M. Xu, J. L. Yao and R. A. Gu, J. Optics-UK, 2015, 17, 114014.

21. G. Baffou. Thermoplasmonics: Heating Metal Nanoparticles Using Light. New York: Cambsidge, 2018.

22. L. K. Khorashad, L. V. Besteiro, Z. Wang, J. Valentine and A. O. Govorov, J. Phys. Chem. C., 2016, 120, 13215-13226.

23. A. O. Govorov, W. Zhang, T. Skeini, H. Richardson, J. Lee and N. A. Kotov, Nanoscale Res. Lett., 2006, 1, 84-90.

24. P. Keblinski, D. G. Cahill, A. Bodapati, C. R. Sullivan and T. A. Taton, J Appl. Phys., 2006, 100, 13549.

25. S. Toroghi, C. Lumdee and P. G. Kik, Appl. Phys. Lett., 2015, 106, 103102.

26. C. L. Baldwin, N. W. Bigelow and D. J. Masiello, J. Phys. Chem. Lett., 2014, 5, 1347-1354

27. W. A. Challener, C. Peng, A. V. Itagi, D. Karns, W. Peng, Y. Peng, X. Yang, X. Zhu, N. J. Gokemeijer, Y. T. Hsia, G. Ju, R. E. Rottmayer, M. A. Seigler and E. C. Gage, Nat. Photonics, 2009, 3, 220-224.

28. J. S. Donner, G. Baffou, D. Mccloskey and R. Quidant, ACS Nano, 2011, 5, 5457-5462.

29. K. Liu, X. Xue and E. P. Furlani, Sci. Rep-UK, 2016, 6, 34189.

30. P. B. Johnson, Phys. Rev. B, 1972, 6, 4370-4379.

31. P. Berto, M. S. A. Mohamed, H. Rigneault and G. Baffou, Phys. Rev. B, 2014, 90, 035439.

32. V. Siahpoush, S. Ahmadi-kandjani and A. Nikniazi, Opt. Commun., 2018, 420, $52-58$.

33. A. Lalisse, G. Tessier, J. Plain and G. Baffou, Sci. Rep-UK, 2016, 6, 38647. 